\title{
Real-time failure detection and identification applied to supervision of oil transport in pipelines
}

\author{
TØRRIS DIGERNES†
}

\begin{abstract}
Keywords: failure detection, failure identification, leakage detection and estimation, multiple hypothesis testing, chi-square test, likelihood ratio tests, multiple model hypothesis probability tests.
\end{abstract}

\begin{abstract}
In this paper methods for real-time failure detection and identification are discussed. The methods apply parallel filters of the Kalman type based on plant models that describe the different failure situations as well as normal operation. A failure is determined by identifying the filter having the highest probability of representing the plant. The tests are based on the innovation sequence produced by each of the filters. The last section presents simulations from a system designed for supervision of oil transport in pipelines.
\end{abstract}

\section{Introduction}

The purpose of a supervision system is to detect and identify, as soon as possible, any failure in the observed process. The information from the process is observed by sensors which may also fail. A sensor failure is not critical, however, if redundant observation systems are used. In the case of no redundancy, the process can still operate in short maintenance periods without complete observation. It is, therefore, important to choose supervision methods capable of discriminating between simple sensor failure and the more serious process failure. Such systems must be devised to avoid production shutdowns by the less critical sensor faults. This is especially important for large and high-cost plants.

A simple and reliable method for detecting sensor failure, which is extensively used, is the local sensor redundancy method with the 'two out of three' voting technique being most common. However, if there are many observation points a large number of sensors would be required which would then create a high global redundancy not properly utilized. If the sensors are complex and expensive the local redundancy method may prove to be unacceptable. Should this be the case, methods using process knowledge to compare different observations from various points within the process may prove to be more feasible.

The most common process failure detection methods are high and/or low alarm limits. These alarm limits are positioned around a normal operating point in the state space or along a normal operating trajectory in the state-time space. These detection methods are usually not applicable or have a reduced reliability during transients, such as load variations, start-up and shut-down periods. Owing to normal fluctuations in the process, the alarm limits are often set far from normal operation bounds to avoid repeated false alarms.

If conventional systems satisfy the design specifications, there is no reason to choose more complicated systems. On the other hand, if more accurate supervision is required, statistical methods and the estimation technique introduced by Kalman 
(1960) may be used. This method is discussed in a paper by Mehra and Peschon (1971). A detailed survey of design methods for failure detection is given by Willsky (1976).

In this paper, some promising methods for failure detection in a supervision system for oil transport are discussed, as well as illustrated, by simulations. A more detailed description may be found in a work report by Digernes (1979).

\section{Formulation of the failure detection problem}

Failure detection may be performed in different ways. There are systems that only give an alarm, systems that identify the failure, or systems that estimate the characteristic values of the failure parameters. In a simple alarm system there is normally not enough information if a quick failure action is required, while a complete estimation of all failure variables normally requires an unacceptable number of sensors to make the model observable. If this is the case, systems based on multiple hypotheses testing seems to be applicable.

The failure identification may be performed by testing the following hypotheses:

$H_{i}(k)$ : The process model $P_{i}$ and the observation model $M_{i}$ represent the plant behaviour at time $k, i=\{1,2, \ldots, K\}$.

One of the hypotheses represents the normal operation, while the others represent the different failure situations.

The tests may be performed by parallel Kalman filters based on the models defined by the hypotheses. The filter innovation, which is the deviation between the plant and the model observation, can then be used to identify the hypothesis having the highest probability of representing the plant.

It is well known that if the plant noise is Gaussian, the Kalman filter innovation also becomes Gaussian with probability density given by

$$
\psi_{i}(k+1 \mid k)=C_{i} \exp \left(0 \cdot 5 r_{i}\left(k+1 \mid k^{\mathrm{T}}\right)^{\mathrm{T}} R_{i}(k+1 \mid k)^{-1} \boldsymbol{r}_{i}(k+1 \mid k)\right)
$$

where $C_{i}=(2 \pi)^{-m_{i} / 2}\left(\operatorname{det} R_{i}(k+1 \mid k)\right)^{-1 / 2} \cdot r_{i}(k+1 \mid k)$ is the innovation at time $k+1$ of the filter based on the $i$ th hypothesis and $R_{i}(k+1 \mid k)$ is the covariance of the innovation. $m_{i}$ is the dimension of the observation vector.

Detailed process failure models are usually not known. However, satisfactory results are normally obtained by simple models of the type $\dot{\boldsymbol{x}}_{\mathrm{F}}=\boldsymbol{v}_{\mathrm{F}}$, where $\boldsymbol{x}_{\mathrm{F}}$ is a vector containing failure parameters in the process model and $\boldsymbol{v}_{\mathrm{F}}$ is white noise.

If redundancy exists in the observations, different observation models may be defined by ordering the sensors in subsets each making the plant observable. To increase the estimation accuracy the normal operating model should contain all sensors. In addition, to prevent the estimation from breakdowns, if all observation models contain failed sensors, there should be an observation model including only noise. A critical situation will only occur if this model is the true observation model, since the estimate is then based on prediction, and process failures are not detectable. However, the process may still operate normally.

A simple observation model that includes failure modes is given by:

$$
y(k)=\mu_{1}\left(h(x(k))+w_{N}\right)+\mu_{2} w_{F}
$$

where $y(k)$ is the observation. In normal operation the failure parameters are given by $\left(\mu_{1}, \mu_{2}\right)=(1,0)$ and the observation noise is assumed to be Gaussian with $w_{\mathrm{N}} \sim N\left(0, W_{\mathrm{N}}\right)$. In the case of failure $\left(\mu_{1}, \mu_{2}\right)=(0,1)$. The failure noise $w_{\mathrm{F}}$ may be 
defined by $w_{\mathrm{F}} \sim N\left(\bar{w}_{\mathrm{F}}, W_{\mathrm{F}}\right)$, normally with $\bar{w}_{\mathrm{F}}=0$, and $W_{\mathrm{F}}>W_{\mathrm{N}}$, but other distributions as, for instance, the uniform distribution may also be used.

\section{Actual test methods}

The failure detection methods most commonly used are the well-known chisquare test and the likelihood ratio test. The first test is mainly an alarm detection method, while the last test is a failure identification method. Since these methods are well known from the literature, only the test variables used shall be defined.

The chi-square test variable is defined by

$$
z_{N i}(k+1)=\sum_{j=k+2-N}^{k+1} r_{i}(i+1 \mid j)^{\mathrm{T}} R_{i}(i+1 \mid j)^{-1} r_{i}(i+1 \mid j)
$$

where index $i$ indicates the $i$ th hypothesis and $N$ is the window of samples used in the test.

The log-likelihood ratio test variable is defined by

$$
\lambda_{i}(k+1)=\ln \left(\psi_{i}(k+1 \mid k) / \psi_{n}(k+1 \mid k)\right)+\lambda_{i}(k)
$$

where $\psi_{i}(k+1 \mid k)$ and $\psi_{n}(k+1 \mid k)$ are the probability density of the innovation from the filters based on the $i$ th failure hypothesis and the normal operation hypothesis, respectively. A detailed description of this method is given by Chien and Adams (1976).

Another promising method which has been tested shall, in this paper, be called the Multiple Model Hypothesis Probability Test (MMHPT). To the author's knowledge, failure detection using this method has not been reported. A detailed description of the method will therefore be given in the next section. The method is, however, closely related to the parallel Kalman filter theory described by Athans and Chang (1976).

\section{The multiple-model hypothesis probability test (MMHP'T)}

This section describes a sequential method that calculates the hypothesis probability for each of the hypotheses. The method is based on a multiple model estimator containing parallel Kalman filters, and algorithms for prediction and updating of the probabilities.

The estimated hypothesis probabilities shall be defined by the following variables:

$\hat{q}_{i}(k+I \mid k)$ : a priori hypothesis probability that hypothesis $H_{i}(k+1)$ is true when the observation sequence up to and including time $k$ is known.

$\hat{q}_{i}(k+1)$ : a posteriori hypothesis probability that hypothesis $H_{i}(k+1)$ is true at time $k+1$ when the observation sequence up to and including time $k+1$ is known.

The relationship between these probabilities, which is derived by Bayes's theorem, is given by

$$
\hat{q}_{i}(k+1)=\frac{\psi_{i}(k+1 \mid k) \hat{q}_{i}(k+1 \mid k)}{\sum_{j=1}^{K} \psi_{j}(k+1 \mid k) \hat{q}_{j}(k+1 \mid k)}, \quad i=\{1,2, \ldots, K\}
$$


where $\psi_{i}(k+1 \mid k)$ is given by eqn. (1), if the innovation is assumed to be approximately Gaussian. The initial values are given by $\hat{q}_{i}(1 \mid 0)=\hat{q}_{0 i}$. Furthermore, we have

$$
\sum_{i=1}^{K} \hat{q}_{i}(k+1)=\sum_{i=1}^{K} \hat{q}_{i}(k+1 \mid k)=1
$$

The prediction from $\hat{q}_{i}(k)$ at time $k$ to $\hat{q}_{i}(k+1 \mid k)$ at time $k+1$ is a processdependent relation which expresses the time evolution of the hypothesis probabilities. Two possible prediction models are

(a) Static prediction with lower bound.

$$
\hat{q}_{i}(k+1 \mid k)=a(k)\left(\left(1-\sigma_{i}\right) q_{\mathrm{Li}}+\sigma_{i} b(k) \hat{q}_{i}(k)\right), \quad i=\{1,2, \ldots, K\}
$$

where $\sigma_{i}=1$ if $\hat{q}_{i}(k)>q_{\mathrm{L} i}$, which is the lower bound of $q_{i}(k)$, otherwise $\sigma_{t}=0$. Further,

$$
b(k)=\left(1-\sum_{i=1}^{K}\left(1-\sigma_{i}\right) q_{\mathrm{L} i}\right) / \sum_{i=1}^{K} \sigma_{i} \hat{q}_{i}(k) \text { and } a(k)=1
$$

A more simple and normally satisfactory prediction is obtained by setting

$$
b(k)=1 \quad \text { and } \quad a(k)=1 / \sum_{i=1}^{K}\left(\left(1-\sigma_{i}\right) q_{\mathrm{L} i}+\sigma_{i} \hat{q}_{i}(k)\right)
$$

(b) Semi-Markov process.

$$
\hat{q}(k+1 \mid k)=Q(k+1 \mid k) \hat{q}(k)
$$

where $\hat{q}(\cdot)^{\mathrm{T}}=\left[\hat{q}_{1}(\cdot), \hat{q}_{2}(\cdot), \ldots, \hat{q}_{K}(\cdot)\right]$ and $Q(K+1 \mid k)$ is a probability transition matrix.

The overall a posteriori state estimate is given by

$$
\begin{aligned}
\hat{x}(k+1) & =E_{x}(x(k+1) \mid Y(k+1)) \\
& =\sum_{i=1}^{K} T_{i} \hat{x}_{i}(k+1) \hat{q}_{i}(k+1)
\end{aligned}
$$

where $Y(k+1)$ is the observation sequence, and $\hat{\boldsymbol{x}}_{i}(k+1)$ is the a posteriori state estimate calculated by the $i$ th filter. $T_{l}$ is a selection matrix, with elements $\{0,1\}$ used to rearrange the elements of $\hat{x}_{i}(\cdot)$ in accordance with the elements of $\hat{x}(\cdot)$. If $\hat{x}(\cdot)$ and $\hat{\boldsymbol{x}}_{i}(\cdot)$ have identical element arrangement, $T_{i}$ becomes an identity matrix.

The overall a posteriori state error covariance is given by

$$
\begin{aligned}
X(k+1) & =E_{x}\left((x(k+1)-\hat{x}(k+1))\left(x(k+1)-\hat{x}(k+1)^{\mathrm{T}} \mid Y(k+1)\right)\right. \\
& =\sum_{i=1}^{K} T_{i}\left(X_{i}(k+1)+\hat{e}_{i}(k+1) \hat{e}_{i}(k+1)^{\mathrm{T}}\right) T_{i}^{\mathrm{T}} \hat{q}_{i}(k+1)
\end{aligned}
$$

where $\hat{\boldsymbol{e}}_{i}(k+1)=\hat{\boldsymbol{x}}_{i}(k+1)-\hat{\boldsymbol{x}}(k+1)$ and $X_{i}(k+1)$ is the error covariance based on the $i$ th filter.

In the case where different observation models are tested, the calculation time may be reduced by the method described in the Appendix.

\section{Simulation examples}

The ability of these methods to detect failure in oil pipeline systems shall be illustrated by simulations. The examples are based on observations generated by a 
pipeline simulator. The pipeline data used are mainly from the Ekofisk-Teesside pipeline.

The pipeline is shown in Fig. 1. The oil streams from the inlet end I to the outlet end $\mathrm{O}$. The pipeline is divided into three $1.0 \times 10^{5} \mathrm{~m}$ sections by the platforms $\mathrm{A}$ and B. The sections are numbered from 1 to 3 counting from the inlet end. The observations are as follows: the inlet flow $w_{1}$; the outlet flow $w_{4}$; the inlet pressure $p_{1}$; the platform pressures $p_{\mathrm{A}}$ and $p_{\mathrm{B}}$; and the outlet pressure $p_{\mathrm{O}}$.

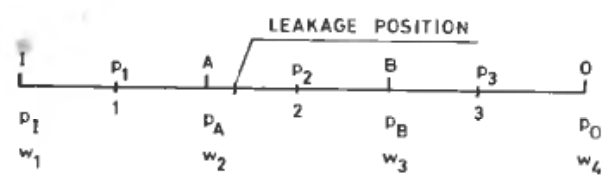

Figure 1. Pipeline structure.

A deterministic process model is given by the following space-discrete equations:

Mass balance

$$
\dot{p}_{i}=\left(w_{i}-w_{i+1}-\theta_{i} w_{\mathrm{L} i}\right) / \kappa_{i}, \quad i=\{1,2, \ldots, s\}
$$

Momentum balance

$$
\left.\begin{array}{rl}
\dot{w}_{1} & =-f_{1}\left(w_{1}\right)+b_{1}\left(p_{1}-p_{1}\right) \\
\dot{w}_{i} & =-f_{i}\left(w_{i}\right)+b_{i}\left(p_{i-1}-p_{i}+\theta_{i-1} p_{\mathrm{L} t-1}\right) \\
\dot{w}_{s+1} & =-f_{s+1}\left(w_{s+1}\right)+b_{s+1}\left(p_{s}-p_{\mathrm{O}}+\theta_{s} p_{\mathrm{L} s}\right)
\end{array}\right\}
$$

External variables

$$
\left.\begin{array}{l}
\dot{p}_{\mathrm{I}}=0 \\
\dot{p}_{\mathrm{O}}=0
\end{array}\right\}
$$

Leakage models

$$
\left.\begin{array}{c}
\dot{w}_{\mathrm{L} i}=0 \\
\dot{p}_{\mathrm{L} i}=0
\end{array}\right\} \quad i=\{1,2, \ldots, s\}
$$

$s$ is the number of sections used in the numerical space discretization, $s=3$ is used in the failure detection program, while $s=15$ is used in the pipeline simulator. The flow states $w_{i}$ and the pressure states $p_{i}$ are defined in Fig. 1 . The failure states $w_{\mathbf{L} i}$ and $p_{\mathrm{L} i}$ are leakage and pressure jumps respectively. The pressure jumps are related to the leakage position and can, together with the leakage estimate, be used to calculate this position. $\theta_{t}$ is a parameter defining the process failure structure in section $i$. $\kappa_{i}, f_{i}(\cdot)$, and $b_{t}$ are compressibility parameters, non-linear friction terms, and pressure force parameters respectively. The temperature accumulation is neglected in this model, but can easily be incorporated by augmenting the model by the energy balance equation. 
The deterministic observation model is given by the following equations:

$$
\begin{aligned}
& y_{1}=w_{1} \\
& y_{2}=w_{\mathrm{O}} \\
& y_{3}=p_{\mathrm{I}} \\
& y_{4}=p_{\mathrm{A}}=\left(p_{1}+\theta_{1} p_{\mathrm{L} 1}+p_{2}\right) / 2 \\
& y_{5}=p_{\mathrm{B}}=\left(p_{2}+\theta_{2} p_{\mathrm{L} 2}+p_{3}\right) / 2 \\
& y_{6}=p_{\mathrm{O}}
\end{aligned}
$$

The noise is defined in accordance with the Kalman filter theory. The following simulated events are used in the tests:

Case A. A $0.3 \mathrm{bbls} / \mathrm{s}$ leakage, which is $1 \%$ of the total flow, occurs at $t=300 \mathrm{~s}$ in the position $1 \cdot 1 \times 10^{5} \mathrm{~m}$ from the inlet end, which is in section 2 .

Case B. As in Case A, but in addition, an abrupt failure occurs in the observation of $p_{\mathrm{B}}$, defined by $p_{\mathrm{B}}=0$ for $t>180 \mathrm{~s}$.

Case C. As in Case A, but now a growing failure occurs in $p_{\mathrm{B}}$, defined by $\overline{\boldsymbol{v}}_{\mathrm{B}}=$ $0.055(t-100)$ for $t>100 \mathrm{~s}$, which is one standard deviation each $100 \mathrm{~s}$.

The methods tested are:

(1) Test of normal operation.

The chi-square test of normal operation is shown in Figs. 2, 3 and 4 representing Cases $\mathrm{A}, \mathrm{B}$ and $\mathrm{C}$ respectively.

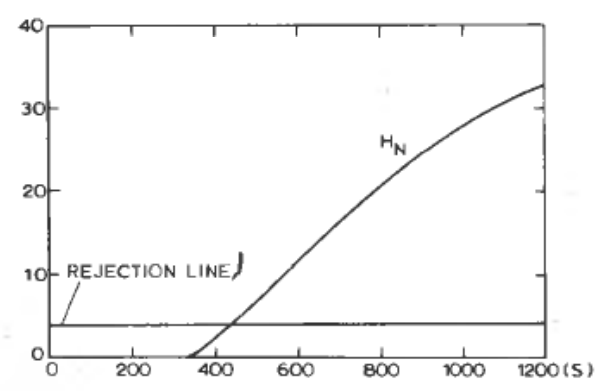

Figure 2. Chi-square test of normal operation, $z_{1}(k)$; Case A.

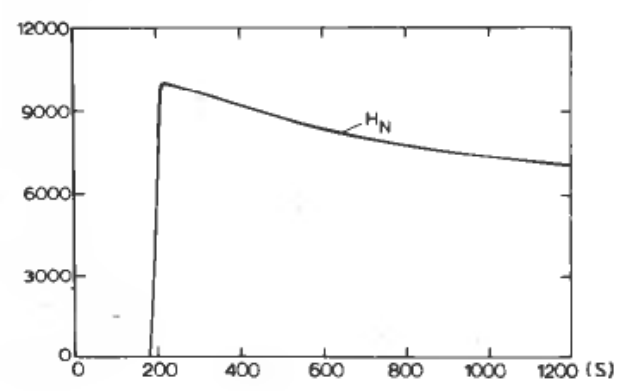

Figure 3. Chi-square test of normal operation, $z_{1}(k)$; Case B. 


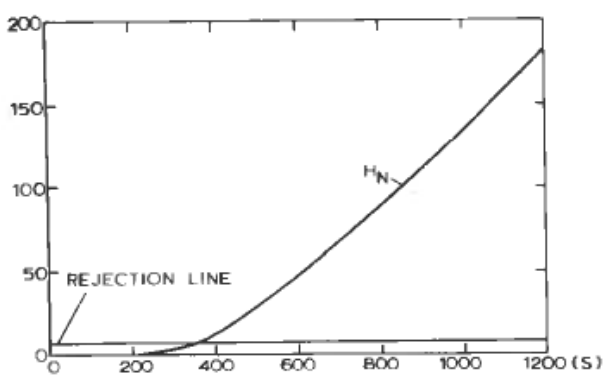

Figure 4. Chi-square test of normal operation, $z_{1}(k)$; Case C.

(2) Failure identification by multiple hypothesis tests.

The following hypotheses are tested:

$$
\begin{aligned}
& H_{\mathrm{N}}: \text { normal operation, } \theta^{\mathrm{T}}=[0,0,0] \\
& H_{\mathrm{L} i}: \text { leakage in section } i, i=\{1,2,3\}, \theta_{j}=\left\{\begin{array}{l}
1 \text { if } i=j \\
0 \text { otherwise }
\end{array}\right. \\
& H_{\mathrm{F} i}: \text { observation failure in sensor } i
\end{aligned}
$$

The sensor failure modes are defined in accordance with the method described in $\S 2$.

The likelihood ratio test is only shown for Case A, Fig. 7. The other tests are all performed by the MMHPT method.

Case A is shown in Figs. 5, 6 and 7. The probability of hypothesis $\mathrm{H}_{\mathrm{N}}$, Fig. 5, increases from the initial value of $0 \cdot 7$ to near $1 \cdot 0$, which indicates that the plant is in normal operation. However, approximately $100 \mathrm{~s}$ after the leakage has occurred, the probability of $H_{\mathrm{N}}$ starts decreasing and a failure is declared about $160 \mathrm{~s}$ after the leakage occurs. The failure is further identified as a leakage in section 2 , hypothesis $H_{\mathrm{L} 2}$, approximately $250 \mathrm{~s}$ after the leakage has occurred. The estimate of the leakage position is $1 \cdot 12 \times 10^{5} \mathrm{~m}$ from the inlet end. This is an accurate result compared with the numerical accuracy used in the space discretization of the process model. A satisfactory estimate of the leakage, Fig. 6, is obtained after approximately $250 \mathrm{~s}$. The log-likelihood ratio test is shown in Fig. 7, where $\max \lambda_{t}=\lambda_{2}$ for $t>560 \mathrm{~s}$ indicates that a leakage has occurred in section 2 .

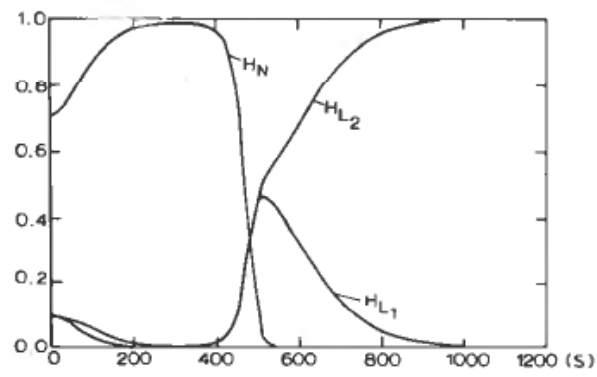

Figure 5. Hypothesis probabilities, $\hat{q}_{l}(k)$; Case A. 


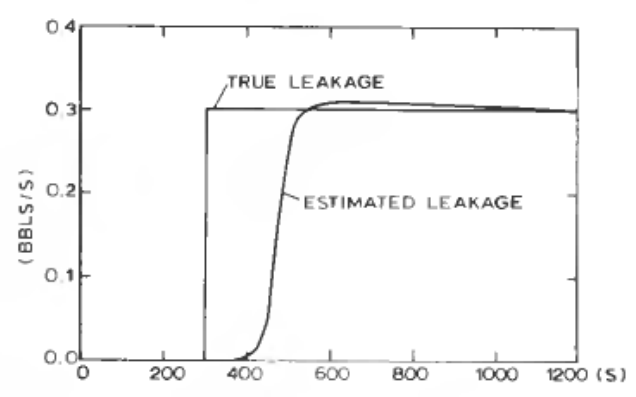

Figure 6. Most probable leakage (bbls/s); Case A.

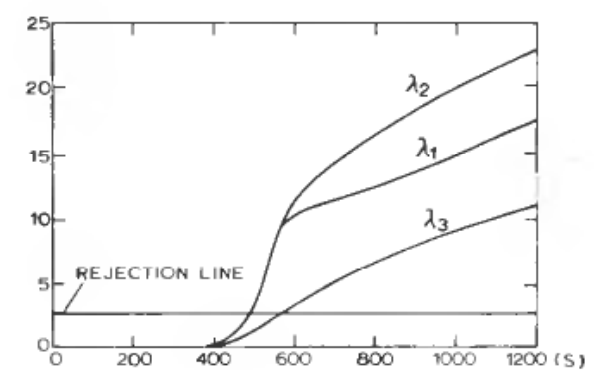

Figure 7. Log-likelihood ratio, $\lambda_{i}(k)$; Case A.

Case B is shown in Figs. 8 and 9. The normal operation hypothesis $H_{\mathrm{N}}$, Fig. 8, is rejected almost immediately after the sensor has failed. At the same time the probability of normal operation and failure in sensor number 5 , indicated by $H_{\mathrm{N}, \mathrm{F} 5}$, in Fig. 8, approaches $1 \cdot 0$. This situation is not dangerous. However, when the leakage occurs, the probability that both a leakage in section 2 and a failure in sensor number 5 , indicated by $H_{\mathrm{L} 2, \mathrm{~F} 5}$ in Fig. 8, approachés $1 \cdot 0$. This is a critical situation since a leakage is detected. A comparison of the leakage estimate in this case, Fig. 9, and Case A, Fig. 6, shows that the estimate differs more from the true leakage in Fig. 9. However, after $400 \mathrm{~s}$ the true leakage and the estimated leakage become approximately identical.

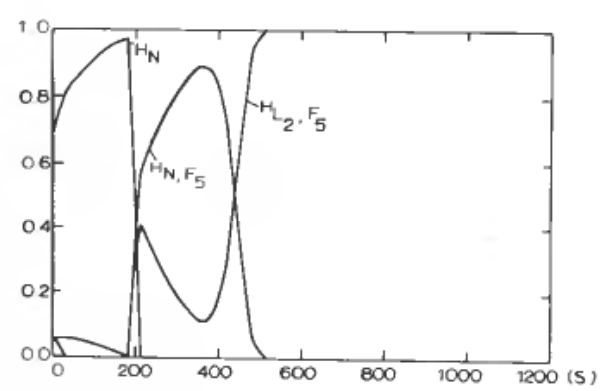

Figure 8. Hypothesis probabilities $\hat{q}_{i}(k)$; Case B. 


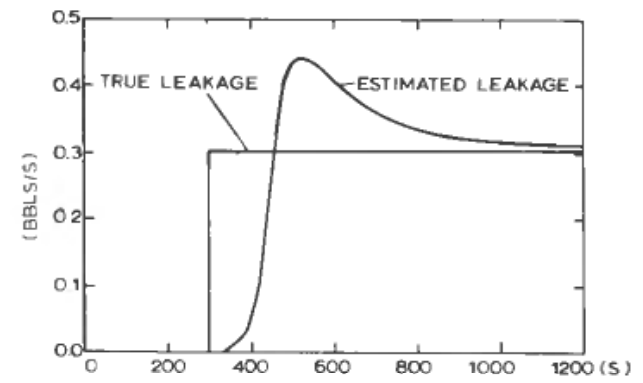

Figure 9. Most probable leakage (bbls/s); Case B.

Case $\mathrm{C}$ is shown in Fig. 10. The leakage estimate is similar to Case B, Fig. 9. The hypothesis probabilities, Fig. 10, are almost identical to case B, Fig. 8 , and a detailed discussion is therefore not repeated.

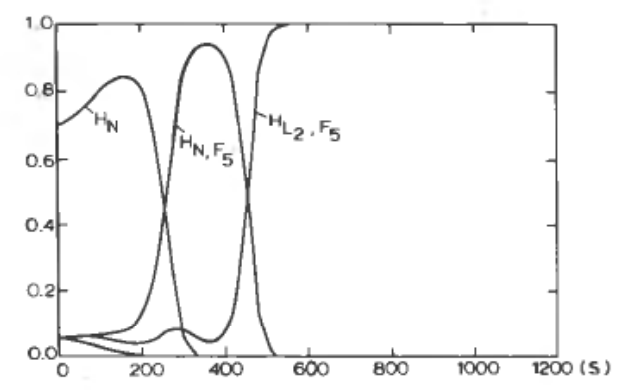

Figure 10. Hypothesis probabilities $\hat{q}_{i}(k)$; Case C.

\section{Conclusion}

In this paper some methods for failure detection have been discussed and illustrated by simulations. The MMHPT method seems to be especially effective for failure identification in oil pipeline systems. This method seems also to give the operator more information concerning the failure situation than the chi-square and the likelihood ratio methods. A practical advantage is that the test variables are normalized to the region (0., 1.). In addition eqn. (9) makes it possible to obtain a better estimate of the failure parameters during transitions from normal to failure operation.

One drawback to methods based on multiple hypothesis testing is that they require relatively large calculation time and storage. However, the MMHPT algorithms are suitable for parallel processing in microprocessors. Another possible solution would be to use a simplified continuous real-time detection method, such as the chi-square test of normal operation, and activate the real-time failure identification only if the chi-square test has detected a failure.

The methods for prediction of the probability density have been tested and the simplest form of eqn. (7) was found to give a satisfactory result in the test cases described.

The method described in the Appendix is not yet completely tested, but seems to give satisfactory results and reduced calculation time if the process models are 
complex and there is a large number of observation models, or if serial processing of the observations is used.

\section{ACKNOWLEDGMENTS}

The simulation examples used are mainly from a report issued by Institutt for Atomenergi and funded by Norpipe A.S. In this report different methods for supervision of the oil pipeline from Ekofisk in the North Sea to Teesside in England were evaluated. The author thanks Norpipe A.S. for their support and for permission to use examples from the report.

\section{Appendix}

Multiple model hypothesis probability test in the case of independent process and observation models

If the process and observation models are independent, the following hypotheses can be tested:

$H_{i j}(k+1)$ : The true hypothesis is a combination of the independent hypotheses $H_{P i}(k+1)$ and $H_{M j}(k+1)$ assuming process model $P_{i}$ and observation model $M_{j}$ to be true; $i=\{1,2, \ldots, I\}, j=\{1,2, \ldots, J\}$.

Owing to the independence, the a posteriori hypothesis probabilities may be split as follows:

$$
\begin{gathered}
\hat{q}_{i j}(k+1 \mid k)=\hat{\alpha}_{i}(k+1 \mid k) \hat{\beta}_{j}(k+1 \mid k) \\
\hat{q}_{i j}(k+1)=\hat{\alpha}_{i}(k+1) \hat{\beta}_{j}(k+1)
\end{gathered}
$$

where $\hat{\alpha}_{t}(\cdot)$ and $\hat{\beta}_{j}(\cdot)$ are the hypothesis probabilities that process model $i$ and observation model $j$ are true.

The a posteriori hypothesis probabilities then become

$$
\begin{aligned}
\hat{\alpha}_{i}(k+1) & =c\left(\sum_{j=1}^{J} \psi_{i j}(k+1 \mid k) \hat{\beta}_{j}(k+1 \mid k)\right) \hat{\alpha}_{i}(k+1 \mid k) \\
\hat{\beta}_{j}(k+1) & =c\left(\sum_{i=1}^{I} \psi_{l j}(k+1 \mid k) \hat{\alpha}_{i}(k+1 \mid k)\right) \hat{\beta}_{j}(k+1 \mid k) \\
c^{-1} & =\sum_{i=1}^{I} \hat{\alpha}_{i}(k+1 \mid k) \sum_{j=1}^{J} \psi_{i j}(k+1 \mid k) \hat{\beta}_{j}(k+1 \mid k)
\end{aligned}
$$

where $\psi_{l \jmath}(k+1 \mid k)$ is the probability density of the innovation based on the $i$ th process model and $j$ th observation model.

The prediction of $\hat{\alpha}_{l}(k+1 \mid k)$ and $\hat{\beta}_{f}(k+1 \mid k)$ may be performed by rules equivalent to those described in $\$ 4$.

The $a$ posteriori state estimates are given by

$$
\begin{aligned}
\hat{\boldsymbol{x}}_{i j}(k+1) & =\hat{\boldsymbol{x}}_{i}(k+1 \mid k)+K_{i j}(k+1) r_{i j}(k+1 \mid k) \\
\hat{\boldsymbol{x}}_{i}(k+1) & =\sum_{j=1}^{J} \hat{\boldsymbol{x}}_{i j}(k+1) \hat{\beta}_{j}(k+1) \\
& =\hat{\boldsymbol{x}}_{i}(k+1 \mid k)+\sum_{j=1}^{J} K_{i j}(k+1) \boldsymbol{r}_{i j}(k+1 \mid k) \hat{\beta}_{j}(k+1) \\
\hat{\boldsymbol{x}}(k+1) & =\sum_{i=1}^{I} T_{i} \hat{\boldsymbol{x}}_{i}(k+1) \hat{\alpha}_{i}(k+1)
\end{aligned}
$$


where $\hat{x}(k+1)$ is the overall a posteriori state estimate. $\hat{\boldsymbol{x}}_{i}(k+1 \mid k)$ and $\hat{\boldsymbol{x}}_{i}(k+1)$ are the $a$ priori state estimate and the a posteriori state estimate based on the $i$ th process model. $\hat{\boldsymbol{x}}_{i j}(k+1), K_{i j}(k+1)$, and $\boldsymbol{r}_{i j}(k+1 \mid k)$ are the $a$ posteriori state estimate, the filter gain, and the innovation based on the $i$ th process model and the $j$ th observation model. $T_{i}$ is a selection matrix belonging to the $i$ th process model.

The $a$ proteriori state error covariance of the $i$ th process model is given by

$$
x_{i}(k+1)=\sum_{j=1}^{J}\left(X_{i j}(k+1)+\hat{\boldsymbol{e}}_{l j}(k+1) \hat{\boldsymbol{e}}_{i j}(k+1)^{\mathrm{T}}\right) \hat{\beta}_{j}(k+1)
$$

where $\hat{e}_{i j}(k+1)=\hat{x}_{i j}(k+1)-\hat{x}(k+1)$, and $x_{i j}(k+1)$ is the a posteriori state error covariance based on the $i$ th process model and the $j$ th observation model.

\section{REFERENCES}

Athans, M., and Chang, C. B. (1976). Adaptive estimation and parameter identification using a multiple model estimation algorithm. Technical note 1976-28, Massachusetts Institute of Technology, Lincoln Laboratory, Lexington, Massachusetts, U.S.A.

Digernes, T. (1979). A discussion and an evaluation of some real-time failure detection and identification methods with application to supervision of oil transport in pipelines. IFA Work Report ESS-11, Institutt for Atomenergi, Kjeller, Norway.

Chien, T. T., and Adams, M. B. (1976). A sequential failure detection technique and its application. I.E.E.E. Trans. autom. Control, 21, 750-757.

Kalman, R. E. (1960). A new approach to linear filtering and prediction problems. $J$. Bas. Engng, 82D, 34-45.

Mehra, R. K., and Peschon, J. (1971). An innovations approach to fault detection and diagnosis in dynamical systems. Automatica 7, 637-640.

WILLSKY, A. S. (1976). A survey of design methods for failure detection in dynamic systems. Automatica, 12, 601-611. 